\title{
Predictors of experiences of discrimination and positive treatment in people with mental health problems: Findings from an Australian national survey
}

Nicola J Reavley, Senior Research Fellow ${ }^{1}$

Amy J Morgan, Research Fellow ${ }^{1,2}$

Anthony F Jorm, Professorial Fellow ${ }^{1}$

${ }^{1}$ Centre for Mental Health, Melbourne School of Population and Global Health, The University of Melbourne, Australia

${ }^{2}$ School of Psychology and Public Health, La Trobe University, Bundoora, Australia

Corresponding author:

Nicola J Reavley, Centre for Mental Health, Melbourne School of Population and Global Health, 207 Bouverie Street, VIC 3010, The University of Melbourne, Australia.

Email: nreavley@unimelb.edu.au, P: +61 390357628 F: +61 393495815

\section{Funding}

The study was funded by the National Health and Medical Research Council. 


\title{
Predictors of experiences of discrimination and positive treatment in
} people with mental health problems: Findings from an Australian national survey

\begin{abstract}
Purpose

The aim of the study was to assess the factors predicting experiences of avoidance, discrimination and positive treatment in people with mental health problems.
\end{abstract}

\section{Methods}

In 2014, telephone interviews were carried out with 5220 Australians aged 18+, 1381 of whom reported a mental health problem or scored highly on a symptom screening questionnaire.

Questions covered experiences of avoidance, discrimination and positive treatment by friends, spouse, other family, workplace, educational institution and others in the community; as well as disclosure of mental health problems. Avoidance, discrimination and positive treatment scores were calculated by counting the number of domains in which each occurred. Predictors of avoidance, discrimination and positive treatment were modelled with negative binomial regression analyses.

\section{Results}

After adjusting for the effects of other predictors in multivariate analyses, symptom severity and a diagnosis of 'any other disorder' (most commonly psychotic disorders or eating disorders) predicted experiences of both avoidance and discrimination but not positive treatment. Disclosing a mental health problem in more settings was also associated with higher rates of avoidance and discrimination, but also with positive treatment. 


\section{Conclusions}

Disclosure of mental health problems to others may increases experiences of discrimination, but may also increase experiences of positive treatment. These findings can help to inform decision making by people with mental health problems about disclosure, particularly in the case of more severe or low-prevalence disorders.

Key words: mental disorders; discrimination; stigma; positive treatment; disclosure 


\section{Background}

Stigma adds to the disability of people with mental health problems. It can contribute to systemic disadvantages in many areas of life, including worsening of psychological distress, inhibition of help seeking and treatment adherence, limiting of personal relationships and reductions in the ability to achieve educational and vocational goals [1-3]. Stigma has been defined by the World Health Organization as "a mark of shame, disgrace or disapproval which results in an individual being rejected, discriminated against, and excluded from participating in a number of different areas of society" [4]. Thornicroft et al. [5] have described stigma as a problem of knowledge: ignorance; a problem of attitudes: prejudice; and a problem of behaviour: discrimination.

Until relatively recently, most stigma-related research explored attitudes towards people with mental health problems, rather than the experiences of people with these problems $[6,7]$. Reviews of evidence about the characteristics of people who are more likely to be stigmatised suggest that greater social distance is desired from people with substance use disorders, followed by schizophrenia and then depression and anxiety disorders [8]. Males elicit greater desire for social distance than females. Similar patterns are seen for beliefs in the dangerousness of people with mental disorders [9]. People who are in treatment are also less likely to be seen as dangerous.

Studies that have attempted to assess experiences of discrimination in people with mental health problems have most often been conducted in clinical populations [10-13], with a small number conducted in the general population [14-17]. Some of these studies have also attempted to explore the factors associated with experiences of discrimination. In a survey of 3570 people using mental health services in England, Hamilton et al. [18] found that age, employment status, length of time in mental health services, disagreeing with the diagnosis, anticipating discrimination in personal relationships and feeling the need to conceal a diagnosis from others were significantly associated with higher levels of experienced discrimination. While there was no direct assessment of symptom severity, the results of this study did show a link between experiences of discrimination and 
involuntary hospital admission, which may be a marker of severity [18]. A recent Canadian study showed that those who reported their mental health as being 'fair' or 'poor' (rather than 'good') were almost three times more likely to have experienced stigma in the preceding 12 months [16]. Other studies in people with both schizophrenia and depression have also shown associations between symptom levels [19], hospitalization and discrimination $[20,12,11]$. Evidence of links between diagnosis and discrimination is inconsistent, with some studies showing greater discrimination experienced by those with a diagnosis of schizophrenia than depression $[12,15]$, while others show the reverse [21] or no difference [19].

Evidence of links between sociodemographic characteristics and experiences of discrimination is also inconsistent. Lower levels of education have been shown to be associated with greater structural discrimination (e.g. that related to health insurance or court proceedings) in patients with schizophrenia and mood and anxiety disorders, while other studies have not found such associations or have tended to report more discrimination in those with higher levels of education $[20,22,12,14,19]$. No consistent association has been found with age or gender and discrimination $[11,12,23]$

Very few studies have examined the links between disclosure and experiences of discrimination and positive treatment. In their multi-country study of discrimination reported by over 1000 people with major depressive disorder, LaSalvia et al. [11] did not find any association between concealment of diagnosis and experience of discrimination once site was taken into account in the analysis. A Dutch study of 500 outpatients in a mental health facility showed that disclosure was negatively correlated with experiences of discrimination and positively correlated with support [24]. The results of a US study of 258 adults with schizophrenia recruited via the internet showed that, although reactions to disclosure varied, many reported worse treatment by police and better treatment by parents after disclosure. Many also experienced worse treatment for medical problems [25]. 
In the context of this inconsistent evidence, the aim of the current study was to use data from an Australian national population-based survey of experiences of avoidance, discrimination and positive treatment in people with mental health problems in order to assess the clinical, sociodemographic and disclosure-related factors associated with these experiences.

\section{Methods}

This paper reports data from a larger study surveying Australians about experiences of discrimination and positive treatment related to mental health problems. For detailed survey methodology see Reavley and Jorm [17]. Briefly, the survey involved computer-assisted telephone interviews (CATI) with a national sample of 5220 members of the general community aged 18 years and over. The sample was contacted by random-digit dialling of both landlines and mobile phones (dual frame design). Interviews were conducted between October and December 2014 by the Social Research Centre survey company. Ethics approval was obtained from the University of Melbourne Human Research Ethics Committee.

\section{Survey interview}

Respondents completed the 12-month version of the Kessler 6 (K6) mental health symptom screening questionnaire [26] and were asked whether they had experienced any sort of mental health problem in the last 12 months. Respondents who answered 'yes' to this question were then asked what they thought the problem was and whether they had received any treatment for it in the last 12 months. For the purposes of the study, respondents were considered to have a mental health problem if they either scored in the high range on the $K 6(>=19)$ or specified any of the following mental health problems: depression/major depression, attempted suicide or self-harm, anxiety/anxiety disorder, post-traumatic stress disorder/PTSD, agoraphobia, panic disorder, obsessive-compulsive disorder/OCD, social phobia, generalised anxiety disorder/GAD, eating disorder/anorexia/bulimia, schizophrenia/paranoid schizophrenia, schizoaffective disorder, 
psychosis/psychotic, bipolar/bipolar disorder/manic-depressive disorder, mental illness, personality disorder/borderline personality disorder, attention deficit-hyperactivity disorder/ADHD, Autism/Asperger's and nervous breakdown. Respondents without a mental health problem were asked questions relating to discrimination against other people with mental health problems (these data are not reported here).

Respondents with a mental health problem were then asked a series of questions about whether they had experienced avoidance, discrimination or more positive treatment because of their mental health problem over the past 12 months across 10 different life domains. These domains were friends, spouse or intimate partner, other members of their family, people in the workplace, when looking for work, people in their place of education, health professionals, other people in the community or neighbourhood, any other people and any other situations. Respondents were told "discrimination occurs when people are treated unfairly because they are seen as being different from others". For the friends domain, respondents were asked "Have any of your friends avoided you because of the emotional or mental health problems you have told me about?"; "Have any of your friends discriminated against you in other ways because of these problems?"; "Have any of your friends treated you more positively because of these problems?" with equivalent wording used for the remaining domains. Further information about the types of experiences reported is covered elsewhere $[27,28]$. The question relating to avoidance was not asked for four domains: 'looking for work', 'health professionals', 'other people' and 'other situations'.

Finally, respondents were asked whether they had disclosed their mental health problem to others in the past 12 months, including friends, intimate partner, supervisor or boss at work, other people in their workplace, teachers or lecturers in their place of education, other people in their place of education, health professionals who were not involved in treating their mental health problem, and people in their neighbourhood or community. 


\section{Statistical analysis}

All analyses were performed using Intercooled Stata 13 (StataCorp LP, Texas, USA). A pre-weight was applied to adjust for the dual frame design and the respondent chance of selection. The achieved sample was close to the Australian national population in terms of geographic distribution, however, there was an under-representation of males and of younger adults, and an over-representation of university-educated individuals and people with an English-speaking background. These biases were adjusted for by 'raking' (also known as rim weighting or iterative proportional fitting) to account for known population proportions of gender, age, education level, region and telephone status.

Avoidance, discrimination and positive treatment scores were calculated by counting the number of domains in which each occurred. Count variables did not adjust for invalid domains, but there were very high correlations between adjusted and unadjusted counts (rs .96-.98). Higher scores therefore indicate more widespread avoidance, discrimination, or positive treatment across life domains. As these variables conformed to a negative binomial distribution, predictors of avoidance, discrimination and positive treatment were modelled with negative binomial regression analyses. Univariate regression analyses were first conducted for each predictor of interest, followed by multiple simultaneous regressions to examine the effect of each predictor adjusting for the effects of other predictors. Sociodemographic predictors were age category; gender; highest level of education; paid or voluntary work outside the home in the past 12 months; marital status; and main language spoken at home. Clinical predictors were categories of psychological distress according to the K6; depression; anxiety disorder (including PTSD and OCD); bipolar disorder; any other disorder (including attempted suicide or self-harm, eating disorder, schizophrenia, schizoaffective disorder, psychosis/psychotic, personality disorder, ADHD, Autism/Asperger's, nervous breakdown, mental illness); whether the person had received treatment for their mental health problem in the past 12 months; and the number of settings in which the person had disclosed their mental health problem (range 0 to 9). Incidence rate ratios (IRRs) report the rate for each predictor at which avoidance, 
discrimination, and positive treatment occurred over the past 12 months. Due to the large number of predictors examined, the $p<.01$ level of significance was used with $99 \%$ confidence intervals.

\section{Results}

Overall, 5220 interviews were completed, with a standard response rate of $37.5 \%$. There were 1381 (28.8\%) respondents classified as having a mental health problem who were asked whether they had experienced avoidance, discrimination, or positive treatment. Of these, 732 respondents had $\mathrm{K} 6$ scores of 19 or above and 1159 respondents had a mental health problem considered to be in scope. Table 1 describes the clinical and sociodemographic characteristics of the sample classified as having a mental health problem.

\section{Avoidance}

There were $43.0 \%$ of respondents who reported being avoided due to their mental health problem in at least one domain. Avoidance scores ranged from 0 to $6(M=0.68, S E=0.03)$. In the univariate analyses, severity (K6 score) was the largest predictor of avoidance scores, along with age, marital status, bipolar disorder, any other disorder, and receiving treatment (see Table 2). Adjusting for the effects of other predictors in the multivariate analysis, severity remained a significant predictor, as did any other disorder. Disclosing a mental health problem in more settings was also associated with higher rates of avoidance in the multivariate analysis. Post-hoc analyses of the 'any other disorder' category were undertaken to explore which of these disorders were specifically associated with avoidance. Psychotic disorder was a significant predictor of experiencing avoidance, IRR $=2.23$ (99\% $\mathrm{Cl}$ : 1.27-3.92), but not after adjusting for other predictors, IRR $=1.77(99 \% \mathrm{Cl}: 0.98-3.20)$. Having an eating disorder was not significantly associated with avoidance in either analysis.

\section{Discrimination}

Discrimination in any life domain was reported by $39.5 \%$ of respondents. Experience of discrimination scores ranged from 0 to $7(M=0.79, S E=0.04)$. The results of the negative binomial 
regression models predicting discrimination are shown in Table 3. Predictors of greater discrimination included age, marital status, severity, any other disorder, received treatment, and count of settings of disclosure. Effects were reduced in the multivariate model, but remained significant for marital status, severity, any other disorder, and count of disclosure. Males also experienced higher rates of discrimination than females in the adjusted analyses. Post-hoc explorations of the 'any other disorder' category found that psychotic disorder was a significant predictor of experiencing discrimination, IRR $=2.32(99 \% \mathrm{Cl}: 1.26-4.27)$, but not after adjusting for other predictors, IRR $=1.80(99 \% \mathrm{Cl}: 0.98-3.33)$. An eating disorder did not predict discrimination in the unadjusted analysis (IRR $=1.63,99 \% \mathrm{Cl}: 0.84-3.17)$, but it did when other predictors were included in the model, IRR $=2.31$ (99\% Cl: 1.14-4.71).

\section{Positive treatment}

The number of settings in which respondents reported positive treatment varied between 0 and 9 , with a mean of $2.25(\mathrm{SE}=0.05)$, and $83.5 \%$ reported positive treatment in at least one domain. As can be seen in Table 4, positive treatment was associated with fewer predictors than for avoidance or discrimination. Those with a bachelor degree experienced lower rates of positive treatment in unadjusted and adjusted analyses. Disclosing a mental health problem in more settings was associated with higher rates of positive treatment in both analyses, while receiving treatment was associated with higher rates of positive treatment in the univariate analysis only.

Avoidance and discrimination scores were highly positively correlated, $r=0.62, p<.001$. Positive treatment scores were weakly correlated with both avoidance $(r=0.12, p<.001)$ and discrimination $(r=0.15, p<.001)$.

\section{Discussion}

This paper reports the results of the first national population-based survey to assess clinical, sociodemographic and disclosure-related factors associated with experiences of avoidance, 
discrimination and positive treatment in people with mental health problems. Results showed that disclosing a mental health problem in more settings was associated with higher rates of both avoidance and discrimination in the multivariate analyses. It was also associated with higher rates of positive treatment. Thus, disclosure leads to both discrimination and support, depending on the circumstances of the individual. This is in line with previous studies in clinical populations, which have reported both positive consequences (e.g. access to treatment, empowerment, improved support, workplace accommodations) and negative consequences (e.g. social isolation and loss of employment opportunities) $[29,24,25]$. These findings reflect the fact that disclosure is likely to depend on context and may not be a black and white decision. Decision aids may be useful for those needing to resolve conflicting needs and values relating to disclosure, particularly in a workplace context [30].

In terms of clinical factors associated with discrimination, after adjusting for the effects of other predictors in multivariate analyses, symptom severity and a diagnosis of 'any other disorder' (most commonly psychotic disorders or eating disorders) predicted experiences of both avoidance and discrimination. In this study, symptom severity was assessed by asking about the worst month in the previous 12 , whereas the question about the type of mental disorder focused on whether the person had ever been given a diagnosis. Thus, it appears that, for someone with a diagnosis of anxiety or depression, people in the social environment respond more to the severity of the person's symptoms and behaviour than to the 'label' of the mental health problem with which they may have been diagnosed. In this study, higher symptom severity was not associated with experiences of positive treatment. Thus, for people with milder problems, positive impact of disclosure might outweigh the negatives, while for a person with more severe problems, the potential for negative consequences is relatively greater. This points to the need to extend anti-stigma education to deepen understanding of the behaviours that someone with less common mental disorders might display and more supportive ways of responding to these behaviours. These findings are in line with 
others that have shown links between discrimination and symptom severity $[16,19]$, as well as hospital admission which may be a marker of severity $[20,12,11]$.

The higher level of discrimination experienced by people with disorders other than depression or anxiety is in line with studies showing people are more likely to hold stigmatizing attitudes towards a person with schizophrenia than to a person with anxiety or depression $[31,32,6]$ and may not be just a function of greater severity of these disorders. While few studies have compared stigma towards people experiencing eating disorders with stigma towards people experiencing other mental disorders, there is some evidence to suggest that stigma towards the former may be greater, particularly in terms of blaming the person for their condition $[33,34]$. The current findings also accord with the findings of other studies on experiences of discrimination, including a 2002 German survey which found that people with schizophrenia reported being avoided twice as often as people with depression [10] and a US study which found higher rates of job-related discrimination in people with schizophrenia compared to people with mood or anxiety disorders [15]. However, other studies have not shown associations between discrimination and a diagnosis of schizophrenia $[18,19]$. As with the findings relating to severity, for people with low-prevalence disorders, the benefits of disclosure may not outweigh the negatives.

Some sociodemographic characteristics (marital status, age and level of education) were associated with discrimination, avoidance and positive treatment in univariate analyses. However, in the multivariate analyses, the only associations remaining for experiences of discrimination were those between lower levels of discrimination and being married. The association between male gender and higher rates of discrimination also remained in multivariate analyses, in line with studies suggesting that males with mental health problems tend to be viewed less favourably $[8,9]$. Having a bachelor degree was associated with lower rates of positive treatment. While no other studies have assessed the impact of level of education on positive treatment, this finding contrasts with other studies which have mostly either shown no associations between experiences of discrimination and 
level of education $[10,16]$, or higher levels of discrimination in people with lower levels of education $[23,20]$.

The study has several strengths. It is the first study to assess, at a population level, mental health problem-related experiences of avoidance, discrimination and positive treatment in a wide range of domains. It was also possible to explore the impact of a wide range of predictive factors, including diagnosis, severity and disclosure. Interviews were used to gather reports of actual experiences of people with a wide range of mental health problems rather than responses to hypothetical scenarios. As a population survey, rather than one that recruited via health services, the results are less likely to be biased towards under-reporting due to people who have experienced very high levels discrimination avoiding service contact [35]. There may also be less bias towards overreporting due to people who have experienced discrimination being more likely to volunteer to take part in a survey on the topic. Limitations of the study include the relatively low response rate of $37 \%$ which, while in line with other similar Australian surveys, may limit the generalisability of the results [36]. In addition, questions only covered voluntary disclosure of mental health problems and analyses have not taken into account the possibility that others may have found out about the person's mental health problems in other ways.

It is hoped that the results of the current study can provide much-needed input into the design of anti-discrimination interventions, for example, public education about the signs and symptoms that someone with depression or anxiety might display and how best to respond to these in a nondiscriminatory, supportive way; decisions about disclosure, particularly as these relate to a person with severe disorders; and how best to manage a situation in which someone discloses a mental health problem.

\section{Funding}

The study was funded by the National Health and Medical Research Council. 


\section{Conflict of interest}

On behalf of all authors, the corresponding author states that there is no conflict of interest.

\section{References}

1. Corrigan P (2004) How stigma interferes with mental health care. Am Psychol 59 (7):614-625

2. Wells JE, Robins LN, Bushnell JA, Jarosz D, Oakley-Browne MA (1994) Perceived barriers to care in St. Louis (USA) and Christchurch (NZ): reasons for not seeking professional help for psychological distress. Soc Psychiatry Psychiatr Epidemiol 29 (4):155-164

3. Link BG, Struening EL, Rahav M, Phelan JC, Nuttbrock L (1997) On stigma and its consequences: evidence from a longitudinal study of men with dual diagnoses of mental illness and substance abuse. J Health Soc Behav 38 (2):177-190

4. World Health Organisation (2001) The World Health Report: Mental health: new understanding, new hope. World Health Organisation, Geneva

5. Thornicroft G, Brohan E, Kassam A, Lewis-Holmes E (2008) Reducing stigma and discrimination: Candidate interventions. Int J Ment Health Syst 2 (1):3. doi:10.1186/1752-4458-2-3

6. Reavley NJ, Jorm AF (2011) Stigmatizing attitudes towards people with mental disorders: Findings from an Australian National Survey of Mental Health Literacy and Stigma. Aust N Z J Psychiatry 48 (12):1086-1093

7. Reavley NJ, Jorm AF (2011) Young people's stigmatizing attitudes towards people with mental disorders: findings from an Australian national survey. Aust N Z J Psychiatry 45 (12):1033-1039. doi:10.3109/00048674.2011.614216

8. Jorm AF, Oh E (2009) Desire for social distance from people with mental disorders. Aust N Z J Psychiatry 43 (3):183-200. doi:908742834 [pii] 10.1080/00048670802653349

9. Jorm AF, Reavley NJ, Ross AN (2012) Belief in the dangerousness of people with mental disorders: A review. Aust N Z J Psychiatry 46 (11):1029-1045. doi:0004867412442406 [pii] $10.1177 / 0004867412442406$ 
10. Angermeyer MC, Beck M, Dietrich S, Holzinger A (2004) The stigma of mental illness: patients' anticipations and experiences. Int J Soc Psychiatry 50 (2):153-162

11. Lasalvia A, Zoppei S, Van Bortel T, Bonetto C, Cristofalo D, Wahlbeck K, Bacle SV, Van Audenhove C, van Weeghel J, Reneses B, Germanavicius A, Economou M, Lanfredi M, Ando S, Sartorius N, LopezIbor JJ, Thornicroft G (2013) Global pattern of experienced and anticipated discrimination reported by people with major depressive disorder: a cross-sectional survey. Lancet 381 (9860):55-62. doi:10.1016/S0140-6736(12)61379-8

12. Thornicroft G, Brohan E, Rose D, Sartorius N, Leese M (2009) Global pattern of experienced and anticipated discrimination against people with schizophrenia: a cross-sectional survey. Lancet 373 (9661):408-415. doi:10.1016/S0140-6736(08)61817-6

13. Wahl OF (1999) Mental health consumers' experience of stigma. Schizophr Bull 25 (3):467-478 14. Alonso J, Buron A, Bruffaerts R, He Y, Posada-Villa J, Lepine JP, Angermeyer MC, Levinson D, de Girolamo G, Tachimori H, Mneimneh ZN, Medina-Mora ME, Ormel J, Scott KM, Gureje O, Haro JM, Gluzman S, Lee S, Vilagut G, Kessler RC, Von Korff M, World Mental Health C (2008) Association of perceived stigma and mood and anxiety disorders: results from the World Mental Health Surveys. Acta Psychiatr Scand 118 (4):305-314. doi:10.1111/j.1600-0447.2008.01241.x

15. Baldwin ML, Marcus SC (2006) Perceived and measured stigma among workers with serious mental illness. Psychiatr Serv 57 (3):388-392. doi:10.1176/appi.ps.57.3.388

16. Stuart H, Patten SB, Koller M, Modgill G, Liinamaa T (2014) Stigma in Canada: results from a rapid response survey. Can J Psychiatry 59 (10 Suppl 1):S27-33

17. Reavley NJ, Jorm AF (2015) Experiences of discrimination and positive treatment in people with mental health problems: Findings from an Australian national survey. Aust N Z J Psychiatry 49 (10):906-913

18. Hamilton S, Corker E, Weeks C, Williams P, Henderson C, Pinfold V, Rose D, Thornicroft G (2016) Factors associated with experienced discrimination among people using mental health services in England. J Ment Health:1-9. doi:10.3109/09638237.2016.1139068 
19. Farrelly S, Clement S, Gabbidon J, Jeffery D, Dockery L, Lassman F, Brohan E, Henderson RC, Williams P, Howard LM, Thornicroft G, group Ms (2014) Anticipated and experienced discrimination amongst people with schizophrenia, bipolar disorder and major depressive disorder: a cross sectional study. BMC Psychiatry 14:157. doi:10.1186/1471-244X-14-157

20. Cechnicki A, Angermeyer MC, Bielanska A (2011) Anticipated and experienced stigma among people with schizophrenia: its nature and correlates. Soc Psychiatry Psychiatr Epidemiol 46 (7):643650. doi:10.1007/s00127-010-0230-2

21. Corker EA, Beldie A, Brain C, Jakovljevic M, Jarema M, Karamustafalioglu O, Marksteiner J, Mohr P, Prelipceanu D, Vasilache A, Waern M, Sartorius N, Thornicroft G, Group FS (2015) Experience of stigma and discrimination reported by people experiencing the first episode of schizophrenia and those with a first episode of depression: The FEDORA project. Int J Soc Psychiatry 61 (5):438-445. doi:10.1177/0020764014551941

22. Dickerson FB, Sommerville J, Origoni AE, Ringel NB, Parente F (2002) Experiences of stigma among outpatients with schizophrenia. Schizophr Bull 28 (1):143-155

23. Alonso J, Buron A, Rojas-Farreras S, de Graaf R, Haro JM, de Girolamo G, Bruffaerts R, Kovess V, Matschinger H, Vilagut G (2009) Perceived stigma among individuals with common mental disorders. J Affect Disord 118 (1-3):180-186. doi:S0165-0327(09)00075-5 [pii] 10.1016/j.jad.2009.02.006 24. Bos AE, Kanner D, Muris P, Janssen B, Mayer B (2009) Mental illness stigma and disclosure: consequences of coming out of the closet. Issues Ment Health Nurs 30 (8):509-513. doi:10.1080/01612840802601382 25. Pandya A, Bresee C, Duckworth K, Gay K, Fitzpatrick M (2011) Perceived impact of the disclosure of a schizophrenia diagnosis. Community Ment Health J 47 (6):613-621. doi:10.1007/s10597-010$9341-1$

26. Kessler RC, Green JG, Gruber MJ, Sampson NA, Bromet E, Cuitan M, Furukawa TA, Gureje O, Hinkov H, Hu CY, Lara C, Lee S, Mneimneh Z, Myer L, Oakley-Browne M, Posada-Villa J, Sagar R, Viana MC, Zaslavsky AM (2010) Screening for serious mental illness in the general population with the K6 
screening scale: results from the WHO World Mental Health (WMH) survey initiative. Int J Methods Psychiatr Res 19 Suppl 1:4-22. doi:10.1002/mpr.310

27. Morgan AJ, Reavley NJ, Jorm AF, Beatson R (2016) Experiences of discrimination and positive treatment from health professionals: A national survey of adults with mental health problems. Aust N Z J Psychiatry 50 (8):754-762. doi:10.1177/0004867416655605

28. Reavley NJ, Morgan AJ, Jorm AF (2016) Discrimination and positive treatment towards people with mental health problems in workplace and education settings: Findings from an Australian national survey. Stigma and Health (in press)

29. Brohan E, Henderson C, Wheat K, Malcolm E, Clement S, Barley EA, Slade M, Thornicroft G (2012) Systematic review of beliefs, behaviours and influencing factors associated with disclosure of a mental health problem in the workplace. BMC Psychiatry 12:11. doi:10.1186/1471-244X-12-11 30. Brohan E, Henderson C, Slade M, Thornicroft G (2014) Development and preliminary evaluation of a decision aid for disclosure of mental illness to employers. Patient Educ Couns 94 (2):238-242. doi:10.1016/j.pec.2013.10.008

31. Angermeyer MC, Matschinger H, Corrigan PW (2004) Familiarity with mental illness and social distance from people with schizophrenia and major depression: testing a model using data from a representative population survey. Schizophr Res 69 (2-3):175-182

32. Lauber C, Nordt C, Falcato L, Rossler W (2004) Factors influencing social distance toward people with mental illness. Community Ment Health J 40 (3):265-274

33. Ebneter DS, Latner JD (2013) Stigmatizing attitudes differ across mental health disorders: a comparison of stigma across eating disorders, obesity, and major depressive disorder. J Nerv Ment Dis 201 (4):281-285. doi:10.1097/NMD.0b013e318288e23f

34. Roehrig JP, McLean CP (2010) A comparison of stigma toward eating disorders versus depression. Int J Eat Disord 43 (7):671-674. doi:10.1002/eat.20760 
35. Clement S, Williams P, Farrelly S, Hatch SL, Schauman O, Jeffery D, Henderson RC, Thornicroft G (2015) Mental health-related discrimination as a predictor of low engagement with mental health services. Psychiatr Serv 66 (2):171-176. doi:10.1176/appi.ps.201300448

36. Curtin R, Presser S, Singer E (2005) Changes in telephone survey non-response over the past quarter century. Public Opin Q 69:87-98

\section{Tables}

Table 1 Sample characteristics

\section{Mental health problem}

Depression

Anxiety (including PTSD, OCD)

Bipolar disorder

Other

Psychotic disorder

Eating disorder

Attempted suicide or self-harm

Personality disorder

Attention deficit-hyperactivity disorder

Autism/Asperger's

Mental illness

\section{Age category}




\section{Gender}

Male

Female

56.4

Highest level of education

Below bachelor level

79.8

Bachelor degree or above

20.2

\section{Employment status}

Paid or voluntary work outside the home in past 12 months

No paid or voluntary work

\section{Marital status}

Married or defacto

Never married

Separated, divorced or widowed

16.0

\section{Language spoken at home}

English

Other

K6 score

6-10 
Told anyone about mental health problem

Yes

95.7

No

Received treatment for mental health problem

Yes

57.5

No

42.5 
Table 2. Predictors of experiencing avoidance related to a mental health problem ${ }^{\mathrm{a}}$

\begin{tabular}{lllll}
\hline \multicolumn{2}{c}{ Univariate } & \multicolumn{2}{c}{ Multivariate } \\
\hline IRR $(99 \% \mathrm{Cl})$ & $\mathbf{p}$ & IRR (99\% CI) & $\mathbf{p}$ \\
Age & & & & \\
$18-29$ & - & - & - & - \\
$30-59$ & $1.01(0.75-1.36)$ & 0.905 & $1.21(0.87-1.67)$ & 0.139 \\
$60+$ & $0.60(0.40-0.91)$ & $\mathbf{0 . 0 0 1}$ & $0.84(0.53-1.34)$ & 0.344
\end{tabular}

\section{Gender}

Male

Female

$0.84(0.65-1.08)$

0.078

$0.82(0.65-1.03)$

0.023

\section{Education}

Below bachelor

Bachelor or above

$0.89(0.69-1.14)$

0.217

$1.02(0.78-1.33)$

0.860

\section{Employment status}

Not working

Working

$0.93(0.71-1.23)$

0.513

$0.96(0.74-1.26)$

0.727

\section{Marital status}

Married or de facto

Never married

$1.46(1.10-1.94)$

0.001

$1.28(0.94-1.75)$

0.036

Separated, divorced or

widowed

$1.41(1.04-1.91)$

0.004

$1.21(0.91-1.62)$

0.081

Language spoken at 


\section{home}

English

Other

$1.24(0.84-1.82)$

0.153

$1.26(0.88-1.82)$

0.100

\section{K6 score}

6-10

11-15

$2.68(1.13-6.38)$

0.003

$2.51(1.11-5.65)$

0.004

16-20

$3.73(1.68-8.27)$

$<0.001$

$3.47(1.60-7.51)$

$<0.001$

21-25

$4.56(2.05-10.13)$

$<0.001$

$4.13(1.91-8.94)$

$<0.001$

26-30

$7.71(3.38$ - 17.59)

$<0.001$

$6.85(3.04-15.43)$

$<0.001$

Disorder ${ }^{b}$

Depression

$1.16(0.89-1.50)$

0.142

$1.03(0.81-1.32)$

0.724

Anxiety disorder

$0.95(0.74-1.22)$

0.592

$0.93(0.74-1.18)$

0.439

Bipolar disorder

$1.58(1.03-2.41)$

0.006

$1.42(0.89-2.26)$

0.053

Any other disorder

$1.85(1.26-2.73)$

$<0.001$

$1.60(1.06-2.40)$

0.003

\section{Received treatment}

No

Yes

$1.43(1.07-1.91)$

0.001

$1.16(0.86-1.55)$

0.198

\section{Count of settings of}

disclosure

$1.07(1.00-1.15)$

0.016

$1.08(1.00-1.15)$

0.006

Note: Number of settings in which respondents reported avoidance ranged from 0 to 6
a. Bold values indicate $p<.01$
b. Each disorder coded as present vs absent
c. $N=1351$ 
Table 3. Predictors of experiencing discrimination related to a mental health problem ${ }^{a}$

\begin{tabular}{|c|c|c|c|c|}
\hline & Univar & & Multiva & \\
\hline & IRR $(99 \% \mathrm{Cl})$ & p & IRR $(99 \% \mathrm{Cl})$ & $p$ \\
\hline Age & & & & \\
\hline $18-29$ & - & - & - & - \\
\hline $30-59$ & $0.99(0.71-1.39)$ & 0.951 & $1.23(0.86-1.76)$ & 0.141 \\
\hline $60+$ & $0.62(0.40-0.95)$ & 0.004 & $0.85(0.51-1.41)$ & 0.403 \\
\hline
\end{tabular}

\section{Gender}

Male

Female

$0.76(0.57-1.00)$

0.011

$0.75(0.59-0.96)$

0.003

\section{Education}

Below bachelor

Bachelor or above

$0.76(0.57-1.01)$

0.014

$0.93(0.70-1.25)$

0.547

\section{Employment status}

Not working

Working

$0.78(0.58-1.05)$

0.032

$0.75(0.56-1.00)$

Marital status

Married or de facto

Never married

$1.68(1.22-2.32)$

$<0.001$

$1.47(1.05-2.06)$

0.003

Separated, divorced or

widowed

$1.77(1.28-2.43)$

$<0.001$

$1.56(1.15-2.11)$

$<0.001$

Language spoken at 


\section{home}

English

Other

$1.16(0.82-1.64)$

0.264

1.31 (0.94 - 1.82)

0.039

\section{K6 score}

6-10

11-15

$1.27(0.62-2.63)$

0.387

$1.20(0.62-2.31)$

0.481

16-20

$1.77(0.91-3.43)$

0.027

$1.64(0.88-3.04)$

0.040

21-25

$2.38(1.21-4.69)$

0.001

$2.06(1.11-3.85)$

0.003

26-30

$4.59(2.31-9.1)$

$<0.001$

$3.80(1.96-7.37)$

$<0.001$

Disorder ${ }^{b}$

Depression

$1.20(0.89-1.61)$

0.114

$1.02(0.79-1.33)$

0.811

Anxiety disorder

$1.18(0.89-1.57)$

0.121

$1.20(0.93-1.56)$

0.065

Bipolar disorder

$1.69(0.86-3.31)$

0.046

$1.60(0.87-2.93)$

0.046

Any other disorder

$2.13(1.47-3.09)$

$<0.001$

$1.89(1.28-2.78)$

$<0.001$

\section{Received treatment}

No

Yes

$1.60(1.16-2.21)$

$<0.001$

$1.17(0.87-1.58)$

0.171

\section{Count of settings of}

disclosure

$1.12(1.03-1.21)$

$<0.001$

$1.15(1.07-1.24)$

$<0.001$

Note: Number of settings in which respondents reported discrimination ranged from 0 to 7
a. Bold values indicate $p<.01$
b. Each disorder coded as present vs absent
c. $\mathrm{N}=1351$ 
Table 4. Predictors of experiencing positive treatment related to a mental health problem ${ }^{\mathrm{a}}$

\begin{tabular}{lllll}
\hline \multicolumn{2}{c}{ Univariate } & \multicolumn{2}{c}{ Multivariate $^{c}$} \\
\hline IRR $(99 \% \mathrm{Cl})$ & $\mathbf{p}$ & IRR (99\% CI) & p \\
Age & & & & \\
$18-29$ & - & - & - & - \\
$30-59$ & $1.04(0.90-1.20)$ & 0.482 & $1.06(0.89-1.26)$ & 0.388 \\
$60+$ & $1.05(0.88-1.25)$ & 0.490 & $1.17(0.94-1.46)$ & 0.071
\end{tabular}

\section{Gender}

Male

Female

$1.12(0.98-1.27)$

0.027

$1.04(0.93-1.18)$

0.342

\section{Education}

Below bachelor

Bachelor or above

$0.87(0.77-0.99)$

0.006

$0.85(0.75-0.96)$

0.001

\section{Employment status}

Not working

Working

$1.07(0.93-1.22)$

0.217

$0.98(0.85-1.14)$

0.752

\section{Marital status}

Married or de facto

Never married

$0.89(0.77-1.02)$

0.027

$0.97(0.83-1.14)$

0.648

Separated, divorced or

widowed

$0.90(0.76-1.06)$

0.097

$0.93(0.80-1.08)$

0.198

Language spoken at 


\section{home}

English

Other

1.09 (0.91 - 1.30)

0.217

$1.13(0.95-1.34)$

0.067

\section{K6 score}

6-10

11-15

$1.02(0.77-1.36)$

0.858

$1.02(0.78-1.33)$

0.859

$16-20$

$1.13(0.87-1.47)$

0.235

$1.11(0.87-1.41)$

0.269

21-25

$1.09(0.83-1.42)$

0.432

$1.10(0.86-1.40)$

0.340

26-30

$1.16(0.87-1.55)$

0.191

$1.15(0.88-1.51)$

0.183

\section{Disorder $^{b}$}

Depression

$1.06(0.93-1.20)$

0.247

$0.99(0.88-1.11)$

0.788

Anxiety disorder

$1.12(0.99-1.26)$

0.018

$1.05(0.93-1.18)$

0.314

Bipolar disorder

$1.09(0.80-1.46)$

0.479

$0.93(0.69-1.25)$

0.533

Any other disorder

$1.05(0.83-1.34)$

0.578

$1.01(0.81-1.27)$

0.887

\section{Received treatment}

No

Yes

$1.25(1.09-1.42)$

$<0.001$

$1.09(0.96-1.25)$

0.081

\section{Count of settings of}

disclosure

$1.19(1.15-1.23)$

$<0.001$

$1.19(1.15-1.23)$

$<0.001$

Note: Number of settings in which respondents reported positive treatment ranged from 0 to 9
a. Bold values indicate $p<.01$
b. Each disorder coded as present vs absent
c. $N=1351$ 


\section{University Library}

\section{- M M I N E R VA \\ A gateway to Melbourne's research publications}

Minerva Access is the Institutional Repository of The University of Melbourne

Author/s:

Reavley, NJ;Morgan, AJ;Jorm, AF

Title:

Predictors of experiences of discrimination and positive treatment in people with mental health problems: findings from an Australian national survey

Date:

2017-03-01

Citation:

Reavley, N. J., Morgan, A. J. \& Jorm, A. F. (2017). Predictors of experiences of discrimination and positive treatment in people with mental health problems: findings from an Australian national survey. SOCIAL PSYCHIATRY AND PSYCHIATRIC EPIDEMIOLOGY, 52 (3), pp.269-277. https://doi.org/10.1007/s00127-016-1301-9.

Persistent Link:

http://hdl.handle.net/11343/123522 